\title{
Rendimiento diagnóstico de tres reglas de predicción clínica para embolia pulmonar
}

\section{The diagnostic yield of three clinical prediction rules for pulmonary embolism}

\author{
Alirio Rodrigo Bastidas-Goyes, Nazhda IVette Faizal-Gómez, \\ Santiago Ortiz-Ramírez, Giuly Aguirre-Contreras • Chía (Colombia)
}

DOI: https://doi.org/10.36104/amc.2020.1384

\section{Resumen}

Objetivo: la embolia pulmonar (EP) es la tercera causa de muerte cardiovascular en el mundo. La evaluación de la probabilidad pre test a través de reglas de predicción clínica Wells, Ginebra y Pisa ha sido ampliamente validada en estudios previos. Sin embargo, hay datos insuficientes que evalúen el rendimiento diagnóstico de las mismas en población colombiana, este artículo tiene como fin evaluar el rendimiento de estas escalas en nuestra población.

Métodos: estudio de cohorte retrospectivo con análisis de prueba diagnóstica en un hospital de III nivel de atención entre los años 2009 y 2017, donde se incluyeron todos los sujetos mayores de 18 años con realización de angiotomografía de tórax (ATC) solicitada por sospecha clínica de EP. Se registraron todas las variables necesarias para la construcción de las reglas de Wells, Ginebra y Pisa. Cada uno de los puntajes se calculó de manera numérica y posteriormente se clasificó según la probabilidad. El diagnóstico de EP se realizó mediante ATC leída por radiólogo. Los datos se ingresaron en una hoja de cálculo de Excel y se analizaron con el programa estadístico SPPS licenciado.

Resultados: se ingresaron 507 sujetos para los puntajes de Wells y Ginebra y 339 para el puntaje de Pisa. El promedio de edad fue de 56 años (DS:19.8) y 56.6\% de sexo masculino, se encontró una relación estadísticamente significativa entre los diferentes puntajes evaluados y el diagnóstico de embolia pulmonar, Wells probabilidad baja, intermedia y alta $\mathrm{p}<0.001$, Wells menos probable y probable $\mathrm{p}<0.001$, Ginebra bajo, intermedio y alto $\mathrm{p}=0.006$, Pisa bajo, intermedio, moderada y alta $\mathrm{p}=0.001$. El ACOR para Wells fue 0.715(IC95\%:0.663-0.767) (p<0.001), Ginebra 0.611(IC95\%:0.553-0.668) $(\mathrm{P}<0.001)$, Pisa 0.643(IC95\%:0.574-0.713) ( $<<0.001)$.

Conclusiones: se determinó un rendimiento superior para el diagnóstico de EP con el puntaje de Wells en nuestro medio, hay limitaciones con la aplicación y desarrollo del puntaje de Pisa asociado a un rendimiento inferior en nuestros pacientes. (Acta Med Colomb 2020; 45. DOI: https://doi. org/10.36104/amc.2020.1384).

Palabras clave: embolismo y trombosis, reproducibilidad y validez, diagnóstico, angiotomografía computarizada de tórax, probabilidad.

\section{Abstract}

Objective: pulmonary embolism (PE) is the third cause of cardiovascular death worldwide. The evaluation of pre-test probability using the Wells, Geneva and Pisa clinical prediction rules has been amply validated in prior studies. However, there are insufficient data for evaluating their diagnostic yield in a Colombian population. The goal of this article is to evaluate the yield of these scales in our population.

Methods: this was a retrospective cohort study with diagnostic test analysis in a tertiary level hospital from 2009 to 2017, which included all subjects over the age of 18 who had undergone a chest computed tomography angiography (CTA) due to a clinical suspicion of PE. All the necessary variables for constructing the Wells, Geneva and Pisa rules were recorded. Each score was calculated numerically and then classified according to probability. Pulmonary embolism was diagnosed through a CTA read by a radiologist. The data were entered on an Excel spreadsheet and analyzed using a licensed SPSS statistical program.
Dr. Alirio Rodrigo Bastidas-Goyes: Médico Internista, Neumólogo, Epidemiólogo; Dres. Nazhda Ivette Faizal-Gómez y Santiago OrtizRamírez: Residentes de Medicina Interna, Dra. Giuly Aguirre-Contreras: Médico General Egresado del Programa de Medicina. Universidad de La Sabana. Chía (Colombia).

Correspondencia: Dr. Alirio Rodrigo BastidasGoyes. Chía (Colombia).

E-mail: alirio.bastidas@unisabana.edu.co Recibido: 16/VI/2019 Aceptado: 10/II/2020 
Results: a total of 507 subjects were included for Wells and Geneva scores and 339 for the Pisa score. The average age was 56 years (SD: 19.8) and 56.6\% were males. A statistically significant relationship was found between the different calculated scores and the diagnosis of pulmonary embolism: low, intermediate and high Wells probability $\mathrm{p}<0.001$; less probable and probable Wells $\mathrm{p}<0.001$; low, intermediate and high Geneva $\mathrm{p}=0.006$; and low, intermediate, moderate and high Pisa $\mathrm{p}=0.001$. The ACOR for Wells was 0.715 (95\% CI:0.663-0.767) $(\mathrm{p}<0.001)$, for Geneva was 0.611 (95\% CI:0.553-0.668) ( $\mathrm{p}<0.001)$, and for Pisa was 0.643 (95\% CI:0.574-0.713) $(\mathrm{p}<0.001)$.

Conclusions: the study showed a greater PE diagnostic yield using the Wells score in our setting. There are limitations to the application and development of the Pisa score asociated with a lower yield in our patients. (Acta Med Colomb 2020; 45. DOI: https://doi.org/10.36104/amc.2020.1384).

Key words: embolism and thrombosis, reproducibility and validity, diagnosis, chest computerized tomography angiography, probability.

\section{Introducción}

La embolia pulmonar (EP) se define como la obstrucción del flujo sanguíneo en los vasos pulmonares generando alteraciones entre la ventilación y perfusión pulmonar con consecuencias potencialmente mortales $(1,2)$. Es una condición que se asocia con alta morbilidad, y que se reconoce como la tercera causa de muerte cardiovascular después del infarto agudo de miocardio y el síndrome neurovascular agudo $(2,3)$. Se estima que aproximadamente un millón de eventos tromboembólicos venosos ocurren cada año en países europeos, donde el $75 \%$ de ellos se deben a EP intrahospitalaria (3). En Colombia no hay muchos datos epidemiológicos de EP, en un estudio realizado por Dennis et al, en 1996 en diferentes hospitales se encontró una prevalencia cercana a $7 \%$ (4).

Desde el desarrollo de la angiotomografía computarizada pulmonar (ATC) como prueba "gold-standard", se ha incrementado un fenómeno de "sobre diagnóstico" de la EP, que según algunos autores ha llevado a encontrar trombos poco significativos y dar tratamiento a pacientes que posiblemente no lo requieren (5). Esto ha generado la necesidad de fortalecer las escalas pre-test con el fin de orientar qué pacientes se benefician de estudios a profundidad (1-3). La ATC es un examen invasivo, costoso y potencialmente riesgoso por lo cual su necesidad debe ser evaluada cuidadosamente (6). Se considera que las reglas de decisión clínica pueden ser comparables al rendimiento diagnóstico de un médico experimentado en el diagnóstico de embolia pulmonar, y son útiles para evitar pruebas innecesarias y potencialmente dañinas sin aumentar el riesgo de infra diagnóstico (7-9).

Existen diferentes escalas o puntajes de predicción clínica para el diagnóstico de EP, los más validados son el puntaje de Wells, Ginebra y Pisa. Si la probabilidad obtenida a través de estas reglas clínicas es alta se recomienda la realización de tomografía computarizada de tórax o gammagrafía de ventilación perfusión pulmonar y si la probabilidad es baja la utilización del dímero D (DD) es útil. La escala de Wells ha sido extensamente validada y dentro nuestra práctica clínica es la más usada $(9,10)$. El cálculo se basa en una correlación entre anamnesis y examen físico que permite calcular una probabilidad baja, media o alta para EP (Wells tradicional) o un resultado probable o poco probable para EP (Wells modificada). Sin embargo, se ha descrito que hasta el $50 \%$ de las veces es omitida o usada de forma incorrecta (11, 12). También parece haber una reducción en la precisión de la escala en pacientes ancianos. Pese a que la escala modificada presenta un rendimiento similar a la escala original, esta última resulta más fácil de correlacionar con los resultados de la gammagrafía ventilación/perfusión $(1,13)$. La escala de Ginebra clasifica los pacientes en tres categorías de probabilidad (baja, intermedia y alta), el puntaje de Pisa puede estimar el riesgo de EP mediante hallazgos clínicos y radiológicos categorizando al paciente de riesgo bajo $(<10 \%)$, intermedio $(>10 \%$ o $<50 \%)$, moderadamente alto $(>50 \%$ y $<90 \%)$ o alto (>90\%) (14). El puntaje de Wells y Ginebra muestran un rendimiento similar para el diagnóstico de embolia pulmonar aguda (15-17), y al parecer el modelo de Pisa parece ser más preciso $(1,18)$. Sin embargo, el rendimiento de estos diferentes puntajes en nuestro medio no es completamente conocido; situación debida en parte a que la utilización y aplicación de estas reglas de predicción clínica en nuestro medio es variable; el conocimiento de las mismas no es uniforme y las variables necesarias para su construcción es diferente.

La dificultad en la toma de decisiones diagnósticas y la elegibilidad de los pacientes en contraste con los costos motivó la realización de este estudio de prueba diagnóstica, que tiene como fin describir el rendimiento diagnóstico de los puntajes de Wells, Ginebra revisado y Pisa con la utilización de radiografía de tórax en los sujetos con sospecha diagnóstica de EP tanto al ingreso a urgencias como en el ambiente hospitalario.

\section{Material y métodos}

Se realizó un estudio de cohorte retrospectivo con análisis de prueba diagnóstica en un hospital de III nivel de atención entre los años 2009-2017, se incluyeron todos los sujetos mayores de 18 años con realización de ATC solicitada por sospecha clínica de EP. Se excluyeron sujetos con realización del examen por sospecha de otras patologías como aneurisma 
de aorta, sospecha de trauma vascular, sospecha de patología aórtica no traumática, síndrome aórtico agudo, sujetos sin información o no localización de la historia clínica por problemas de sistema de información.

Se registraron todas las variables clínicas y paraclínicas necesarias para la construcción de los puntajes de Wells, Ginebra revisado y Pisa con hallazgos radiográficos, siguiendo la recomendación de los autores para la construcción de cada una de estos puntajes al momento de la sospecha diagnóstica de la EP $(19,20)$. Se incluyeron de manera independiente las variables de edad y sexo, lugar de sospecha de la enfermedad (urgencias u hospitalización), historia de enfermedad cardiovascular, antecedente de infarto agudo de miocardio (IAM), falla cardiaca, hipertensión arterial (HTA), fibrilación auricular, valvulopatía, enfermedad pulmonar (EPOC, ASMA, fibrosis pulmonar), antecedentes de cirugía en las últimas cuatro semanas, utilización de anestesia general, antecedente de trombosis venosa profunda, embolia pulmonar, trauma en las últimas cuatro semanas, fractura de miembros inferiores, malignidad, cáncer activo en el último año, tratamiento de malignidad, utilización de anticonceptivos orales; hallazgos clínicos de inmovilización mayor de tres días, disnea, tiempo de disnea, dolor torácico, hemoptisis, signos y síntomas de trombosis venosa profunda, diagnóstico más probable embolia pulmonar, estado mental, temperatura, presión arterial sistólica, presión arterial diastólica, frecuencia cardiaca, frecuencia respiratoria, saturación arterial de oxígeno $\left(\mathrm{SaO}_{2}\right)$, hallazgos ECG de sobrecarga ventricular derecha (VD), hallazgos radiológicos de oligohemia pulmonar, amputación arterial hiliar, consolidación con o sin infarto pulmonar, DD el diagnóstico de EP por ATC (21).

Cada uno de los puntajes se calculó de manera numérica y posteriormente se clasificó según la probabilidad; el puntaje de Wells en tres niveles (baja: $<2$, intermedia: 2-6 y alta: $>6$ ) $(10,22)$ y dos niveles (menos probable $\leq 4$ y probable $>4$ ) (23), el puntaje de Ginebra revisado en tres niveles (bajo: 0-3, intermedio: 4-10, alto 11-22) (15)y el puntaje de Pisa en cuatro niveles (baja: 0-10, intermedio: 11-50, moderada alta: 51-80 y alta 81-100) (1). El diagnóstico de EP se realizó con el resultado de la ATC leída por radiólogo como estudio positivo para embolia pulmonar $(8,24)$.

El tamaño de muestra se calculó tomando los resultados del metaanálisis de Lucassen W (19), donde se encontró para la escala de Wells una sensibilidad de 0.85 y especificidad de 0.51 y para el puntaje de Ginebra revisada una sensibilidad de 0.91 y especificidad de 0.37 , para el cálculo de intervalo de confianza con una prevalencia de la enfermedad de $30 \%$, precisión 5\% y nivel de confianza del $95 \%$ un mínimo de 503 sujetos, se ingresaron los sujetos con un muestreo a conveniencia y secuencial hasta completar el número requerido.

Los datos se ingresaron posterior a una hoja de cálculo de Excel y posteriormente se analizaron con el programa estadístico SPPS licenciado, las variables cualitativas se resumieron en frecuencias y porcentajes y las cuantitativas según su distribución, si era normal en promedio y desviación estándar y si era no normal en mediana y rango intercuartílico, se realizó un análisis bivariado con cada una de las variables estudiadas, las variables cuantitativas se compararon según su distribución con $t$ de student o $\mathrm{U}$ de Mann Whitney y las cualitativas con chi ${ }^{2}$. Una vez construidos los puntajes, se compararon con el diagnóstico o no de EP mediante tomografía y posteriormente se construyó y realizó un análisis del área bajo la curva de características operativas del receptor (ACOR) con cada uno de los puntajes cuantitativos de las reglas de predicción clínica calculando el intervalo de confianza del $95 \%$ y considerándose una $\mathrm{p}$ estadísticamente significativa $<0.05$. Se siguieron las recomendaciones éticas de Helsinki y resolución 8430 de 1993 para investigación en humanos, así como la reserva y protección de datos.

\section{Resultados}

Se ingresaron al análisis final 507 sujetos para los puntajes de Wells y Ginebra y 339 para el puntaje de Pisa. En la Figura 1 se muestra el ingreso de los sujetos al estudio. La EP se encontró en $24.8 \%$ de todos los sujetos evaluados. En la Tabla 1 se muestran las características sociodemográficas y antecedentes de la población y la relación con EP. Se encontró una relación estadísticamente significativa entre el antecedente de HTA y EP ( $\mathrm{p}=0.023)$. En la Tabla 2 se muestran las características clínicas y hallazgos al examen físico de la población. Incluye los síntomas clínicos como el dolor torácico, la hemoptisis, la presencia de manifestaciones clínicas de trombosis venosa profunda y los signos vitales (la temperatura, frecuencia cardiaca y frecuencia respiratoria), cuya positividad mostró una relación estadísticamente significativa compatible con el hallazgo de EP $(\mathrm{p}<0.05)$.

El electrocardiograma (ECG) fue realizado a 404 (79.5\%) de los sujetos, sin encontrarse una relación estadísticamente significativa entre el reporte de sobrecarga del VD y la presencia de $\mathrm{EP}(\mathrm{p}=0.567)$. Por otro lado, la radiografía de tórax se realizó en 463 (91.3\%) de los sujetos, encontrándose qué hallazgos de oligohemia y consolidación se relacionan con el diagnóstico tomográfico. El DD se realizó en 122 (43.5\%) de los sujetos, encontrándose niveles superiores en los pacientes con embolia pulmonar. En la Tabla 3 se muestran las características de los hallazgos ECG, radiológicos y de DD en la población de estudio.

Se encontró una relación estadísticamente significativa con las diferentes reglas de predicción clínica y el diagnóstico de EP, a mayores puntajes mayor proporción de sujetos con hallazgos en la ATC de EP. El puntaje de Pisa, con un número menor de sujetos para su construcción, mostró también diferencias significativas para discriminar sujetos con y sin EP. En la Tabla 4 se resumen los hallazgos mencionados en relación con los puntajes evaluados y el diagnóstico de EP.

El puntaje de Wells mostró un área bajo la curva de características operativas del receptor (ROC, por sus siglas en inglés) mayor a los puntajes de Ginebra revisado y de Pisa en la población de estudio. Los valores detallados de 
Tabla 1. Características Sociodemográficas y antecedentes en la población.

\begin{tabular}{|c|c|c|c|c|}
\hline & Población total $\mathbf{n = 5 0 7}$ & Sin EP $n=381$ & Con $E P n=126$ & Valor $\mathbf{p}$ \\
\hline Edad años x(ds) & $56(19.8)$ & $56.4(19.7)$ & $54.9(20.3)$ & 0.527 \\
\hline Sexo masculino $\mathrm{n}(\%)$ & $287(56.6)$ & $156(40.9)$ & $64(50.8)$ & 0.053 \\
\hline Sospecha de EP en hospitalización n(\%) & $122(24.1)$ & $87(22.8)$ & $35(27.8)$ & 0.26 \\
\hline Historia de enfermedad cardiovascular n(\%) & $124(24.5)$ & $89(23.4)$ & $35(27.8)$ & 0.317 \\
\hline IAM & $19(3.7)$ & $14(3.7)$ & $5(4)$ & 0.88 \\
\hline Falla cardíaca & $36(7.1)$ & $27(7.1)$ & $9(7.1)$ & 0.983 \\
\hline HTA & $188(37.1)$ & $152(39.9)$ & $36(28.6)$ & 0.023 \\
\hline Fibrilación auricular & $25(4.9)$ & $22(5.8)$ & $3(2.4)$ & 0.127 \\
\hline Valvulopatía & $16(3.2)$ & $11(2.9)$ & $5(4)$ & 0.547 \\
\hline Historia de enfermedad pulmonar n(\%) & $122(24.1)$ & $97(25.5)$ & $25(19.8)$ & 0.201 \\
\hline EPOC & $64(12.6)$ & $48(12.6)$ & $16(12.7)$ & 0.977 \\
\hline Asma & $12(2.4)$ & $10(2.6)$ & $2(1.6)$ & 0.507 \\
\hline Fibrosis pulmonar & $6(1.2)$ & $6(1.6)$ & $0(0)$ & 0.156 \\
\hline \multicolumn{5}{|l|}{ Antecedentes n (\%) } \\
\hline Cirugías últimas cuatro semanas & $145(28.6)$ & $110(28.9)$ & $35(27.8)$ & 0.814 \\
\hline Anestesia general cirugía & $113(22.3)$ & $88(23.1)$ & $25(19.8)$ & 0.279 \\
\hline Antecedente de TVP & $41(8.1)$ & $29(7.6)$ & $12(9.5)$ & 0.495 \\
\hline Antecedente de EP & $39(7.7)$ & $21(5.5)$ & $18(14.3)$ & 0.001 \\
\hline Trauma últimas cuatro semanas & $69(13.6)$ & $56(14.7)$ & $13(10.3)$ & 0.214 \\
\hline Fractura MI & $41(8.1)$ & $34(8.9)$ & $7(5.6)$ & 0.229 \\
\hline Antecedente malignidad & $52(10.3)$ & $39(10.2)$ & $13(10.3)$ & 0.979 \\
\hline Cáncer activo último año & $30(5.9)$ & $19(5)$ & $11(8.7)$ & 0.123 \\
\hline Tratamiento malignidad & $17(3.4)$ & $10(2.6)$ & $7(5.6)$ & 0.113 \\
\hline Tratamiento paliativo malignidad & $4(0.8)$ & $2(0.5)$ & $2(1.6)$ & 0.243 \\
\hline Uso ACO & $7(1.4)$ & $4(1)$ & $3(2.4)$ & 0.267 \\
\hline
\end{tabular}

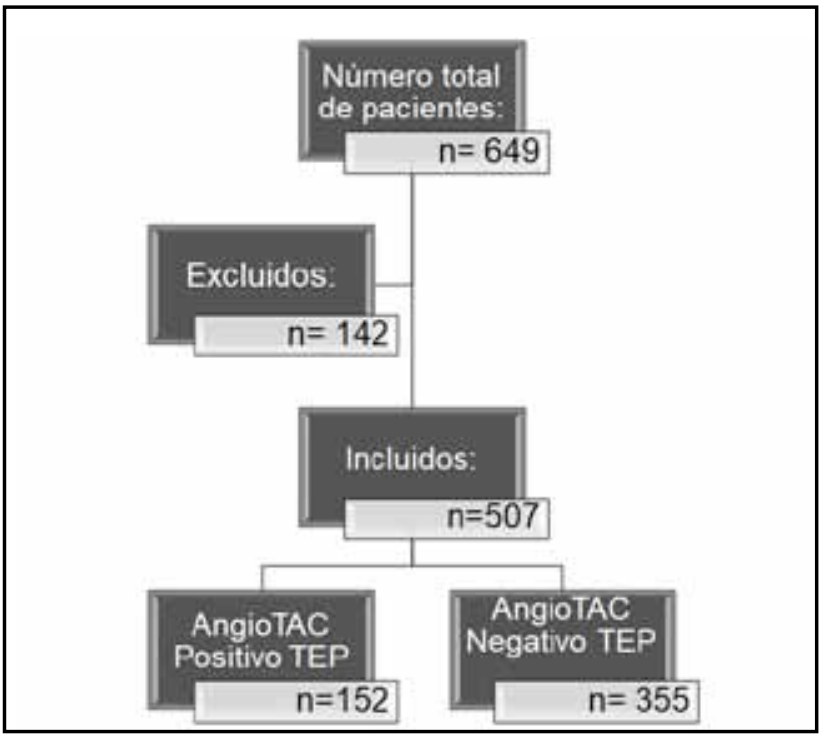

Figura 1. Materiales y métodos.. las diferentes áreas ROC se muestran en la Tabla 5. Estos valores se mantienen en análisis con el mismo número de sujetos donde se pudieron construir las tres reglas de predicción clínica de manera completa.

\section{Discusión}

Según la literatura encontrada, este es el primer estudio en nuestro medio que evalúa el rendimiento diagnóstico de tres reglas de predicción clínica para el diagnóstico de embolia pulmonar. Toma sujetos de los servicios de urgencias y hospitalización encontrando que el mayor rendimiento diagnóstico es con el puntaje de Wells (25); no obstante, nuestros hallazgos son diferentes a lo encontrado por Miniati et al (14), quienes en una cohorte con características similares de 1100 pacientes y una prevalencia de EP del $40 \%$, encuentran un alto rendimiento diagnóstico para el puntaje de Pisa (18). Si bien, el puntaje de Pisa puede ser más preciso para el diagnóstico de EP en ciertos contextos clínicos, ya que utiliza un mayor número de variables, tanto 
Tabla 2. Características clínicas y hallazgos al examen físico en la población.

\begin{tabular}{|c|c|c|c|c|}
\hline & Población total n=507 & $\begin{array}{c}\text { Sin EP } \\
\mathrm{n}=381\end{array}$ & $\begin{array}{c}\text { Con EP } \\
n=126\end{array}$ & $\begin{array}{c}\text { Valor } \\
\mathbf{p}\end{array}$ \\
\hline \multicolumn{5}{|l|}{ Hallazgos clínicos n (\%) } \\
\hline Inmovilización > tres días & $90(17.8)$ & $62(16.3)$ & $28(22.2)$ & 0.13 \\
\hline Disnea aguda & $380(75)$ & $287(75.3)$ & $93(73.8)$ & 0.733 \\
\hline Tiempo disnea M (rango) & $1(0-30)$ & $1(0-30)$ & $2.3(0-21)$ & 0.577 \\
\hline Dolor torácico & $311(61.3)$ & $221(58)$ & $90(71.4)$ & 0.007 \\
\hline Hemoptisis & $30(5.9)$ & $18(4.7)$ & $12(9.5)$ & 0.048 \\
\hline Dolor MI unilateral & $63(12.4)$ & $41(10.8)$ & $22(17.5)$ & 0.037 \\
\hline Edema unilateral & $60(11.8)$ & $35(9.2)$ & $25(19.8)$ & 0.001 \\
\hline Dolor palpación MI & $58(11.4)$ & $36(9.4)$ & $22(17.5)$ & 0.014 \\
\hline Signos y síntomas de TVP & $47(9.3)$ & $25(6.6)$ & $22(17.5)$ & $<0.001$ \\
\hline Diagnóstico más probable de EP & $250(49.3)$ & $151(39.6)$ & 99 (78.6) & $<0.001$ \\
\hline \multicolumn{5}{|l|}{ Estado mental n (\%) } \\
\hline Desorientación & $5(1)$ & $5(1.3)$ & $0(0)$ & 0.445 \\
\hline Letargia & $7(1.4)$ & $6(1.6)$ & $1(0.8)$ & \\
\hline Estupor & $4(0.8)$ & $4(1.1)$ & $0(0)$ & \\
\hline Coma & $15(3)$ & $12(3.2)$ & $3(2.4)$ & \\
\hline Alerta & $473(93.8)$ & $352(92.9)$ & $121(96.8)$ & \\
\hline \multicolumn{5}{|l|}{ Hallazgos examen físico $x(d s)$} \\
\hline Temperatura ${ }^{\circ} \mathrm{C}$ & $36.6(0.69)$ & $36.7(0.7)$ & $36.5(0.7)$ & 0.022 \\
\hline PAS mmHg & $121(20)$ & $122.5(20.5)$ & $119.7(18.6)$ & 0.196 \\
\hline PAD mmHg & $72.9(14)$ & $73.1(14.7)$ & $72.1(11.9)$ & 0.965 \\
\hline FC lpm & $92(19.3)$ & $91.3(19.1)$ & $94.9(19.7)$ & 0.038 \\
\hline FR rpm & $21(5.7)$ & $20.8(5.4)$ & $21.7(6.5)$ & $<0.001$ \\
\hline $\mathrm{SaO} 2 \%$ & $89.2(7.4)$ & $89.1(7.2)$ & $89.4(7.7)$ & 0.368 \\
\hline
\end{tabular}

Tabla 3. Características de los hallazgos electrocardiográficos (ECG), radiológicos (Rx) y Dímero D de la población.

\begin{tabular}{|c|c|c|c|c|}
\hline & ECG $n=403$ & Sin EP n=297 & Con EP $n=106$ & Valor $\mathbf{p}$ \\
\hline \multirow[t]{2}{*}{ Sobrecarga VD n(\%) } & $40(9.9)$ & $28(9.4)$ & $12(11.3)$ & 0.567 \\
\hline & Rx de tórax $n=463$ & Sin EP n=347 & Con EP n=116 & Valor $\mathrm{p}$ \\
\hline Oligohémia & $6(1.3)$ & $2(0.6)$ & $4(3.4)$ & 0.018 \\
\hline Amputación arterial hiliar & $2(0.4)$ & $1(0.3)$ & $1(0.9)$ & 0.415 \\
\hline Consolidación & $81(17.5)$ & $61(17.6)$ & $20(17.2)$ & 0.934 \\
\hline Consolidación infarto pulmonar & $14(3.3)$ & $4(1.3)$ & $10(9.6)$ & $<0.001$ \\
\hline Consolidación no infarto pulmonar & $66(15.6)$ & $55(17.4)$ & $11(10.5)$ & 0.093 \\
\hline \multirow[t]{2}{*}{ Edema pulmonar } & $16(3.5)$ & $14(4)$ & $2(1.7)$ & 0.237 \\
\hline & Dímero D n=221 & Sin EP $n=166$ & Con EP n=55 & Valor $\mathrm{p}$ \\
\hline Resultado dímero $\mathrm{D} x(\mathrm{ds})$ & $3(2.7)$ & $2.5(2.3)$ & $4.7(3.3)$ & $<0.001$ \\
\hline
\end{tabular}


Alirio Rodrigo Bastidas-Goyes y cols.

Tabla 4. Resultados de los puntajes de Wells, Ginebra revisado, Pisa con criterio radiológico y diagnóstico de EP.

\begin{tabular}{|c|c|c|c|c|}
\hline & Población total $\mathrm{n}=507$ & $\operatorname{Sin} \mathbf{E P} n=381$ & Con $\mathbf{E P} n=126$ & Valor $\mathbf{p}$ \\
\hline \multicolumn{5}{|c|}{ Puntaje de Wells probabilidad tres niveles n (\%) } \\
\hline Baja $<2$ & $205(40.4)$ & $183(48)$ & $22(17.5)$ & $<0.001$ \\
\hline Intermedia 2-6 & $216(42.6)$ & $159(41.7)$ & $57(45.2)$ & \\
\hline Alta $>6$ & $86(17)$ & $39(10.2)$ & $47(37.3)$ & \\
\hline \multicolumn{5}{|c|}{ Puntaje de Wells probabilidad dos niveles n(\%) } \\
\hline Menos probable $\leq 4$ & $298(58.8)$ & $255(66.9)$ & $43(34.1)$ & $<0.001$ \\
\hline Probable $>4$ & $209(41.2)$ & $126(33.1)$ & $83(65.9)$ & \\
\hline \multicolumn{5}{|c|}{ Puntaje de Ginebra revisado n (\%) } \\
\hline Bajo 0-3 & $142(28)$ & $117(30.7)$ & $25(19.8)$ & 0.006 \\
\hline Intermedio 4-10 & $324(63.9)$ & $240(63)$ & $84(66.7)$ & \\
\hline \multirow[t]{2}{*}{ Alto $11-22$} & $41(8.1)$ & $24(6.3)$ & $17(13.5)$ & \\
\hline & Población total n=339 & $\operatorname{Sin} E P n=253$ & Con $\mathrm{EP} \mathrm{n}=86$ & \\
\hline \multicolumn{5}{|c|}{ Puntaje de Pisa con radiografía de tórax n (\%) } \\
\hline Baja 0-10 & $113(44.7)$ & $22(25.2)$ & $135(39.8)$ & 0.001 \\
\hline Intermedia 11-50 & $127(50.2)$ & $50(58.1)$ & $177(52.2)$ & \\
\hline Moderada alta 51-80 & $11(4.3)$ & $10(11.6)$ & $21(6.2)$ & \\
\hline Alta $81-100$ & $2(0.8)$ & $4(4.7)$ & $6(1.8)$ & \\
\hline
\end{tabular}

Tabla 5. Área bajo la curva de COR de los puntajes de Wells, Ginebra revisado y Pisa con radiografía de tórax para el diagnóstico de EP en la población total y discriminado en pacientes en urgencias y hospitalización.

\begin{tabular}{|c|c|c|c|}
\hline & Total & ACOR (IC95\%) & Valor $\mathbf{p}$ \\
\hline Wells & 507 & $0.715(0.663-0.767)$ & $<0.001$ \\
\hline Ginebra & 507 & $0.611(0.553-0.668)$ & $<0.001$ \\
\hline Pisa & 339 & $0.643(0.574-0.713)$ & $<0.001$ \\
\hline \multicolumn{4}{|c|}{ Urgencias } \\
\hline Wells & 385 & $0.722(0.662-0.783)$ & $<0.001$ \\
\hline Ginebra & 385 & $0.612(0.545-0.678)$ & 0.001 \\
\hline Pisa & 261 & $0.670(0.594-0.746)$ & $<0.001$ \\
\hline \multicolumn{4}{|c|}{ Hospitalización } \\
\hline Wells & 122 & $0.701(0.600-0.802)$ & 0.001 \\
\hline Ginebra & 122 & $0.606(0.493-0.719)$ & 0.068 \\
\hline Pisa & 78 & $0.568(0.414-0.721)$ & 0.354 \\
\hline & & & \\
\hline
\end{tabular}

Tabla 6. Área bajo la curva de COR de los puntajes de Wells, Ginebra revisado y Pisa con radiografía de tórax para el diagnóstico de EP en la población total y discriminado en pacientes en urgencias y hospitalización.

\begin{tabular}{|c|c|c|c|}
\hline & Total & ACOR (IC95\%) & Valor $\mathbf{p}$ \\
\hline Wells & 507 & $0.715(0.663-0.767)$ & $<0.001$ \\
\hline Ginebra & 507 & $0.611(0.553-0.668)$ & $<0.001$ \\
\hline Pisa & 339 & $0.643(0.574-0.713)$ & $<0.001$ \\
\hline \multicolumn{4}{|c|}{ Urgencias } \\
\hline Wells & 385 & $0.722(0.662-0.783)$ & $<0.001$ \\
\hline Ginebra & 385 & $0.612(0.545-0.678)$ & 0.001 \\
\hline Pisa & 261 & $0.670(0.594-0.746)$ & $<0.001$ \\
\hline \multicolumn{4}{|c|}{ Hospitalización } \\
\hline Wells & 122 & $0.701(0.600-0.802)$ & 0.001 \\
\hline Ginebra & 122 & $0.606(0.493-0.719)$ & 0.068 \\
\hline Pisa & 78 & $0.568(0.414-0.721)$ & 0.354 \\
\hline & & & \\
\hline
\end{tabular}


clínicas como paraclínicas que modifican la probabilidad EP (1). El bajo rendimiento en nuestro estudio pudo verse influenciado por la evaluación de la radiografía de tórax y ECG que pueden ser interpretados dependiendo de la experticia del evaluador, no obstante, de ser correcta esta afirmación el puntaje de Pisa presentaría una desventaja con relación a Wells o Ginebra en nuestro medio dado que estos últimos puntajes podrían ser construidos con mayor facilidad (13).

En nuestros hallazgos, el puntaje de Wells es superior al de Ginebra, aun utilizando criterios subjetivos para el mismo, lo que favorece y facilita el abordaje de nuestros pacientes para el diagnóstico de esta condición. Lucassen et al, en un metaanálisis limitado por heterogeneidad, encuentra una sensibilidad entre 60 y $85 \%$ y una especificidad entre 51 y $80 \%$ a diferentes puntos de corte de Wells, siendo superior el puntaje de Ginebra en sensibilidad con un porcentaje entre 84 y $91 \%$, pero inferior en valores de especificidad la cual se encontró entre 37 y $50 \%$ en este estudio $(19,26)$. La escala Wells también demostró mayor validez que la escala de Ginebra en un estudio de cohorte de 203 pacientes hospitalizados con disnea o dolor torácico, en donde se compararon ambas escalas y se documentó una sensibilidad y especificidad del 79-90\% para la escala de Wells, y de 66$51 \%$ para la escala revisada de Ginebra $(22,25)$.

Entre otros hallazgos en nuestro estudio, encontramos que variables como el antecedente previo de TEP (5.5\% vs $14.2 \% \mathrm{p}<0.001)$, hallazgos clínicos compatibles con TVP (6.6\% vs $17.5 \%, \mathrm{p}<0.001)$, considerar que el diagnóstico más probable es EP (39.6\% vs $78.6 \%$, p<0.001), la consolidación leída como infarto pulmonar en la radiografía, el DD elevado y la taquipnea $(5.4 \%$ vs $6.5 \%$, p <0.001), concuerdan en diferencias estadísticamente significativas encontradas en los estudios originales de los puntajes estudiados, sin embargo, el antecedente de malignidad, la inmovilización $>3$ días, oligohemia en placa de tórax, el dolor torácico y la hemoptisis mostraron una tendencia a la asociación estadística $(27,28)$, situación que podría estar en relación con diferencias poblacionales; sin embargo, el análisis general de los puntajes es satisfactorio (12).

Estudios recientes apuntan a que el rendimiento de la ATC se potencia si se usan escalas clínicas previo a la realización de la misma $(2,6)$, esto sugiere que la probabilidad de obtener un resultado positivo para EP después de la prueba diagnóstica depende no sólo de la sensibilidad y especificidad del test "per se" si no también de la probabilidad clínica previa a la realización de la prueba confirmatoria (24). Además, utilizar la ATC como patrón de referencia permite también descartar diagnósticos alternativos como la neumonía, cuyos hallazgos pueden estar presentes entre el 8 y $22 \%$ de pacientes sometidos a ATC. Valores de DD menores son útiles para descartar la EP cuando la probabilidad clínica es baja, en nuestros hallazgos el DD es menor en los sujetos con ATC negativa $(8,29,30)$.

Entre las debilidades de este estudio se puede contemplar la naturaleza retrospectiva del mismo, donde se puede tener un subregistro de variables clínicas necesarias para el cálculo de todos los puntajes, especialmente para el de Ginebra y Pisa. Puede contemplarse un sesgo de espectro de enfermedad al evaluarse pacientes en un nivel de atención de alta complejidad, ya que pueden ingresar los más graves y sintomáticos, si bien, se utilizó la ATC para evaluar el diagnóstico final, es necesario conocer que hasta el $2 \%$ de los pacientes con resultado negativo de ATC pueden tener embolia pulmonar dentro de los 90 días posteriores y en poblaciones de bajo riesgo se pueden obtener resultados positivos entre 6 y $10 \%$ (8). Este estudio abre la puerta a nuevas investigaciones y a futuro se podrían realizar estudios adicionales evaluando el dímero D y la escala PERC en pacientes con baja probabilidad de EP en nuestro medio con poblaciones de mayor tamaño, así como evaluar el impacto económico en nuestros centros de atención $(8,29,31)$. Finalmente no se estudiaron poblaciones especiales como mujeres embarazadas, poblaciones con TEP de alto riesgo, expectativas de vida limitada y población pediátrica donde estas reglas de predicción clínica pueden tener rendimiento diferente $(7,32)$.

\section{Conclusión}

Se encontró un rendimiento mayor para el diagnóstico de EP con el puntaje de Wells en nuestro medio, hay limitaciones con la aplicación y desarrollo del puntaje de Pisa asociado a un rendimiento inferior en nuestros pacientes. Los resultados podrían ser corroborados con poblaciones mayores y no ser extrapolables en poblaciones especiales como mujeres embarazadas, poblaciones con TEP de alto riesgo y pacientes con expectativa de vida limitada

\section{Referencias}

1. Cronin P, Dwamena BA. A Clinically Meaningful Interpretation of the Prospective Investigation of Pulmonary Embolism Diagnosis (PIOPED) Scintigraphic Data. Acad Radiol. 2017;24(5):550-62.

2. Cronin P, Dwamena BA. A Clinically Meaningful Interpretation of the Prospective Investigation of Pulmonary Embolism Diagnosis (PIOPED) II and III Data. Acad Radiol. 2018;25(5):561-72.

3. Righini M, Gal GL, Bounameaux H. Approach to Suspected Acute Pulmonary Embolism: Should We Use Scoring Systems? Semin Respir Crit Care Med. 2017;38(1):3-10.

4. Dennis R, Rodríguez MN. Estudio nacional sobre tromboembolismo venoso en población hospitalaria en Colombia. Acta Med Colomb. 1996;21:55-63.

5. Wiener RS, Schwartz LM, Woloshin S. Time trends in pulmonary embolism in the United States: evidence of overdiagnosis. Arch Intern Med. 2011;171(9):831-7.

6. Stein PD, Hull RD. Multidetector computed tomography for the diagnosis of acute pulmonary embolism. Curr Opin Pulm Med. 2007;13(5):384-8.

7. Biss TT, Brandao LR, Kahr WH, Chan AK, Williams S. Clinical probability score and D-dimer estimation lack utility in the diagnosis of childhood pulmonary embolism. J Thromb Haemost. 2009;7(10):1633-8.

8. Freund Y, Cachanado M, Aubry A, Orsini C, Raynal PA, Feral-Pierssens AL, et al. Effect of the Pulmonary Embolism Rule-Out Criteria on Subsequent Thromboembolic Events Among Low-Risk Emergency Department Patients: The PROPER Randomized Clinical Trial. JAMA. 2018;319(6):559-66.

9. van Belle A, Buller HR, Huisman MV, Huisman PM, Kaasjager K, Kamphuisen PW, et al. Effectiveness of managing suspected pulmonary embolism using an algorithm combining clinical probability, D-dimer testing, and computed tomography. JAMA. 2006;295(2):172-9.

10. Yap KS, Kalff V, Turlakow A, Kelly MJ. A prospective reassessment of the utility of the Wells score in identifying pulmonary embolism. Med J Aust. 2007;187(6):333-6. 
11. Newnham M, Stone H, Summerfield R, Mustfa N. Performance of algorithms and pre-test probability scores is often overlooked in the diagnosis of pulmonary embolism. BMJ. 2013;346:f1557.

12. Geersing GJ, Erkens PM, Lucassen WA, Buller HR, Cate HT, Hoes AW, et al. Safe exclusion of pulmonary embolism using the Wells rule and qualitative D-dimer testing in primary care: prospective cohort study. BMJ. 2012;345:e6564.

13. Bass AR, Fields KG, Goto R, Turissini G, Dey S, Russell LA. Clinical Decision Rules for Pulmonary Embolism in Hospitalized Patients: A Systematic Literature Review and Meta-analysis. Thromb Haemost. 2017;117(11):2176-85.

14. Miniati M, Monti S, Bottai M. A structured clinical model for predicting the probability of pulmonary embolism. Am J Med. 2003;114(3):173-9.

15. Klok FA, Mos IC, Nijkeuter M, Righini M, Perrier A, Le Gal G, et al. Simplification of the revised Geneva score for assessing clinical probability of pulmonary embolism. Arch Intern Med. 2008;168(19):2131-6.

16. Le Gal G, Righini M, Roy PM, Sanchez O, Aujesky D, Bounameaux H, et al. Prediction of pulmonary embolism in the emergency department: the revised Geneva score. Ann Intern Med. 2006;144(3):165-71

17. Robert-Ebadi H, Mostaguir K, Hovens MM, Kare M, Verschuren F, Girard P, et al. Assessing clinical probability of pulmonary embolism: prospective validation of the simplified Geneva score. J Thromb Haemost. 2017;15(9):1764-9.

18. Miniati M, Bottai M, Monti S. Comparison of 3 clinical models for predicting the probability of pulmonary embolism. Medicine (Baltimore). 2005;84(2):107-14.

19. Lucassen W, Geersing GJ, Erkens PM, Reitsma JB, Moons KG, Buller H, et al. Clinical decision rules for excluding pulmonary embolism: a meta-analysis. Ann Intern Med. 2011;155(7):448-60.

20. Ceriani E, Combescure C, Le Gal G, Nendaz M, Perneger T, Bounameaux $\mathbf{H}$, et al. Clinical prediction rules for pulmonary embolism: a systematic review and meta-analysis. J Thromb Haemost. 2010;8(5):957-70.

21. Douma RA, Mos IC, Erkens PM, Nizet TA, Durian MF, Hovens MM, et al. Performance of 4 clinical decision rules in the diagnostic management of acute pulmonary embolism: a prospective cohort study. Ann Intern Med. 2011;154(11):70918.

22. Wells PS, Ginsberg JS, Anderson DR, Kearon C, Gent M, Turpie AG, et al. Use of a clinical model for safe management of patients with suspected pulmonary embolism. Ann Intern Med. 1998;129(12):997-1005.
23. Schouten HJ, Geersing GJ, Oudega R, van Delden JJ, Moons KG, Koek HL. Accuracy of the Wells clinical prediction rule for pulmonary embolism in older ambulatory adults. J Am Geriatr Soc. 2014;62(11):2136-41.

24. Fabia Valls MJ, van der Hulle T, den Exter PL, Mos IC, Huisman MV, Klok FA. Performance of a diagnostic algorithm based on a prediction rule, D-dimer and CT-scan for pulmonary embolism in patients with previous venous thromboembolism. A systematic review and meta-analysis. Thromb Haemost. 2015;113(2):406-13

25. Di Marca S, Cilia C, Campagna A, D'Arrigo G, Abd ElHafeez S, Tripepi G, et al. Comparison of Wells and Revised Geneva Rule to Assess Pretest Probability of Pulmonary Embolism in High-Risk Hospitalized Elderly Adults. J Am Geriatr Soc. 2015;63(6):1091-7.

26. Lucassen WA, Erkens PM, Geersing GJ, Buller HR, Moons KG, Stoffers HE, et al. Qualitative point-of-care D-dimer testing compared with quantitative D-dimer testing in excluding pulmonary embolism in primary care. J Thromb Haemost. 2015;13(6):1004-9.

27. Chunilal SD, Eikelboom JW, Attia J, Miniati M, Panju AA, Simel DL, et al. Does this patient have pulmonary embolism? JAMA. 2003;290(21):2849-58.

28. Wang RC, Bent S, Weber E, Neilson J, Smith-Bindman R, Fahimi J. The Impact of Clinical Decision Rules on Computed Tomography Use and Yield for Pulmonary Embolism: A Systematic Review and Meta-analysis. Ann Emerg Med. 2016;67(6):693-701.e3.

29. Kearon C, de Wit K, Parpia S, Schulman S, Afilalo M, Hirsch A, et al. Diagnosis of Pulmonary Embolism with d-Dimer Adjusted to Clinical Probability. $N$ Engl J Med. 2019;381(22):2125-34.

30. van der Hulle T, Cheung WY, Kooij S, Beenen LFM, van Bemmel T, van Es J, et al. Simplified diagnostic management of suspected pulmonary embolism (the YEARS study): a prospective, multicentre, cohort study. Lancet. 2017;390(10091):289-97.

31. Singh B, Parsaik AK, Agarwal D, Surana A, Mascarenhas SS, Chandra S. Diagnostic accuracy of pulmonary embolism rule-out criteria: a systematic review and meta-analysis. Ann Emerg Med. 2012;59(6):517-20.e1-4.

32. Agha BS, Sturm JJ, Simon HK, Hirsh DA. Pulmonary embolism in the pediatric emergency department. Pediatrics. 2013;132(4):663-7. 